\title{
Monitoring Low-Cycle Fatigue Material-Degradation by Ultrasonic Methods
}

\author{
R. Himawan ${ }^{1^{*}}$, H. Katoh ${ }^{2}$ and A. Cahyono ${ }^{3}$ \\ ${ }^{I}$ Center for Reactor Technology and Nuclear Safety, National Nuclear Energy Agency, \\ Puspiptek, Serpong, Tangerang 15314, Indonesia \\ ${ }^{2}$ Department of Mechanical Engineering, Saitama University, Shimo-Okubo 255, Sakura-ku Saitama-shi, 338-8570, Japan \\ ${ }^{3}$ Center for Nuclear Intrumentation Engineering, National Nuclear Energy Agency \\ Puspiptek, Serpong, Tangerang 15314, Indonesia
}

\section{ARTICLE INFO}

Article history:

Received 20 November 2009

Received in revised form 31 August 2010

Accepted 31 August 2010

Keywords:

Low-cycle fatigue

Non-destructive test

Ultrasonic method

\begin{abstract}
A B S T R A C T
Any system consisting of structural material often undergoes fatigue, which is caused by dynamic load cycle. As a structural system, nuclear power plant is very likely to have low-cycle fatigue at many of its components. Taking into account the importance of monitoring low-cycle fatigue on structural components to prevent them from getting failure, the authors have conducted a work to monitor material degradation caused by low-cycle fatigue by using ultrasonic method. An alloy of $\mathrm{Cu}-40 \mathrm{Zn}$ was used as a test specimen. Ultrasonic water immersion procedure was employed in this ultrasonic test. The probe used is a focusing type and has frequency as high as $15 \mathrm{MHz}$. The specimen area tested is in the middle part divided into 14 points $\times 23$ points. The results, which were frequency spectrums, were analyzed using two parameters: frequency spectrum peak intensity and attenuation function gradient. The analysis indicates that peak intensity increases at the beginning of load cycle and then decreases. Meanwhile, gradient of attenuation function is lower at the beginning of fatigue process, and then consistently gets higher. It concludes that low-fatigue material degradation can be monitored by using ultrasonic method.
\end{abstract}

(C) 2010 Atom Indonesia. All rights reserved

\section{INTRODUCTION}

Structural material in service commonly receives cyclic load. This cyclic load can make material experience fatigue, which may lead to fracture. Based on the number of load cycle causing fatigue fracture, fatigue can be classified into low cycle and high cycle. Low-cycle fatigue refers to less than 10,000 times of load cycle that generates fracture (due to high enough stress resulting plastic deformation), while high-cycle fatigue relates to more than 10,000 times of load cycle to cause fracture[1].

Like any other structural systems, nuclear power plant (NPP) is subject to either low-or high-cycle fatigue. Many structural components of NPP, such as reactor pressure vessel, primary cooling pipe, pressurizer, main isolating valves, and re-circulating pumps, undergo low-cycle fatigue. High-cycle fatigue commonly occurs at pumps and tubes of heat exchanger.

Material fatigue consists of two processes: before and after crack initiation. Before-crack

\footnotetext{
* Corresponding author.

E-mail address: roziqh@yahoo.com (R. Himawan)
}

initiation process includes plastic deformation and slip band in micro structure. In the next process, fatigue continues and results in crack propagation, which then causes fracture. To prevent fatigue fracture from taking place in components of NPP while in operation, an in-service inspection program is conducted by using non-destructive tests, one of which is ultrasonic method. Considering that fatigue phenomenon may result in material failure, it is crucial that material degradation caused by fatigue be monitored.

Ching, et.al performed monitoring to micro structure which experienced plastic deformation with various strain quantity using ultrasonic method. Wherever, plastic deformation is the beginning step of crack initiation[2]. The results shown that ultrasonic wave parameters have been changing relating to the quantity of plastic deformation. In this study, ultrasonic method is employed to monitor material degradation caused by low-cycle fatigue.

It is expected that the results of this work may give benefit to in-service inspection program in order to ensure structural component integrity of NPP. 


\section{THEORY}

\section{Fatigue in Metal}

Fatigue is defined as changes in mechanical properties, which may take place in metal because of the presence of periodical stress or strain acting on it. These changes eventually may lead to material fracture or rupture. When a load is acted on a metal, the metal will experience elastic deformation along with the increase in stress up to its yield strength $\left(\sigma_{y s}\right)$. Further increase in stress, it will undertake plastic deformation. Even though, metal material receives stress smaller than its yield strength, fatigue process has been initiated by local plastic deformation, which later may cause small fracture propagating to form bigger fracture and, finally, result in rupture. Plastic deformation initiating fatigue process is made possible by the presence of cyclic load.

Plastic deformation occurs since material structure has slip planes, which will deform if load stress is present. Different direction of load stress makes different slips, i.e. extrusion and intrusion. Continuous and periodic load stress makes intrusion slips become micro cracking, which then propagates to generate macro cracking.

\section{Non-Destructive Test}

In-service inspection (ISI) is performed to guarantee that the structure integrity of an operating installation is maintained well. The ISI activities should not provide negative impact on mechanical properties of the supporting components of the system of interest. Therefore, ISI usually employs methods that will not give any damage to the tested objects. There are several non-destructive methods used widely, one of which is ultrasonic method.

As indicated by its name, this method utilizes ultrasonic wave for the test. A pulser, an electronic circuit, is used to generate ultrasonic wave in the form of electric energy, which is then converted into mechanical energy and transferred to test specimen by a sensor called probe. When an ultrasonic wave hits inhomogeneous characteristics of acoustic, this wave will be reflected and caught by the probe, and then changed back to electric energy. Nowadays, ultrasonic method is used to detect any defect that might occur on fabricated material. This method is also able to estimate young modulus, metal hardness, porosity, etc.

A study carried out by Ching et al. showed that parameters of ultrasonic testing correlate with deformation quantity on metal material.[2] Therefore, based on the results of that study, ultrasonic method is used to monitor material degradation caused by low-cycle fatigue.

In ultrasonic testing, the results obtained are in the form of reflected wave, transmission wave or scattered wave signals, which are in time domain. In this study, the results of ultrasonic testing are analyzed in frequency domain. To transform time domain into frequency domain, Fourier Transform is used. If $f(t)$ is time domain function, and $F(\omega)$ is frequency domain function, then Fourier Transform is expressed as [2]

$$
F(\omega)=\int_{-\infty}^{\infty} f(t) e^{-j \omega t} d t
$$

where $t$ is time, $\omega$ is frequency, and $j$ is imaginary number.

To perform the analysis, ultrasonic signal is digitized to obtain numerical data. Since $F(\omega)$ is a continuous function, it is necessary to have a discrete function. Equation 1 is expressed as a discrete function[2]

$$
f_{j}=\sum_{k=0}^{n-1} x_{k} e^{-\frac{2 \pi i}{n} j k}
$$

where $x$ is numerical data of the results of ultrasonic testing and $\mathrm{n}$ is number of data. An algorithm to calculate Equation 2 is called Fast Fourier Transform (FFT) and the most common one is Cooley-Tukey algorithm. An example of ultrasonic signal and frequency spectrum is shown in Fig. 1.

To do the quantification, two parameters used are spectrum peak intensity and attenuation function gradient. This ultrasonic testing employs two data of reflected wave signal taken from surface reflected wave and back wall reflected wave of the specimen. The comparison between frequency spectrum of reflected wave of upper surface, $I_{R}(\omega)$, and of lower surface, $I_{B}(\omega)$, is expressed by Equation 3 [2].

$$
\Gamma(\omega)=20 \times \log _{10}\left(\frac{I_{R}(\omega)}{I_{B}(\omega)}\right)
$$

This equation is called transfer function and also known as attenuation function [2]. Furthermore, in order to obtain gradient of attenuation function, regression in a range of $10-20 \mathrm{MHz}$ is needed as shown in Fig. 2. 


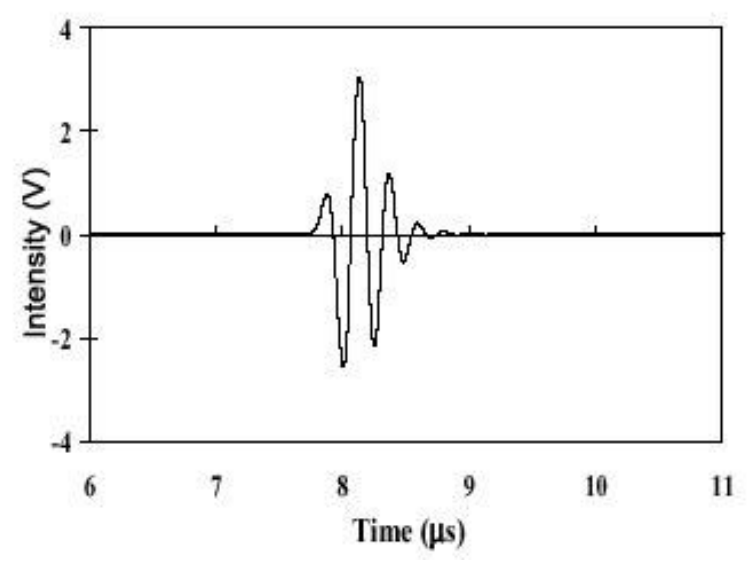

(a) Reflected Wave Signal

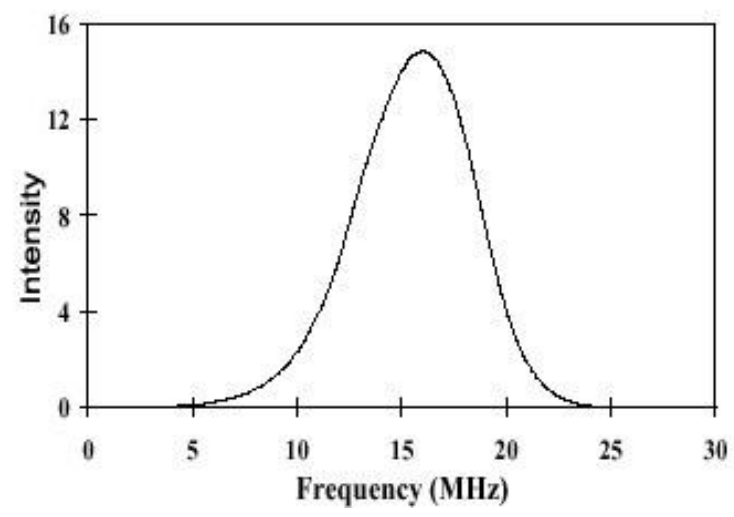

(b) Frequency Spectrum of Reflected Wave

Fig. 1. Example of Ultrasonic Signal and Frequency Spectrum Resulted from FFT.

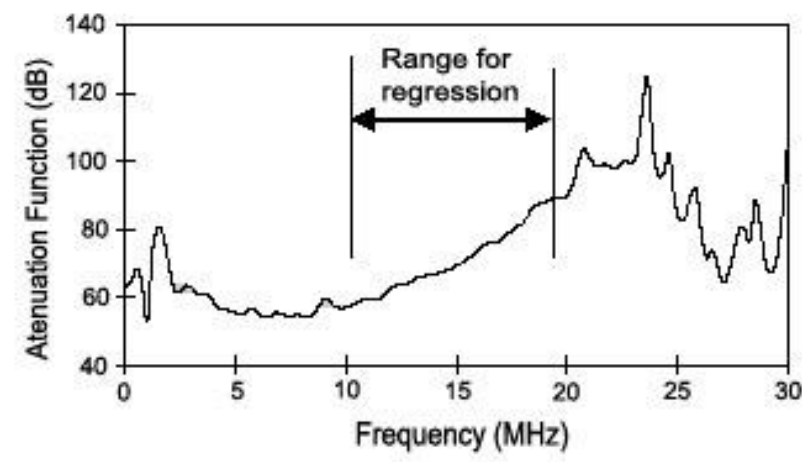

Fig. 2. Example of Attenuation Function.

\section{EXPERIMENTAL METHODS}

\section{Material}

Specimen used in this study is a plate of $\mathrm{Cu}-40 \mathrm{Zn}$ alloy having thickness of $4 \mathrm{~mm}$. The composition of this alloy is shown in Table 1. Fig. 3 depicts its shape and dimension. The middle part has curved shape with the radius of $100 \mathrm{~mm}$ to make concentrated stress and to allow plastic deformation and fracture in this area.
Table 1. Composition of Alloy $\mathrm{Cu}-40 \mathrm{Zn}$ (wt $\%$ )

\begin{tabular}{cccc}
\hline $\mathrm{Cu}$ & $\mathrm{Pb}$ & $\mathrm{Fe}$ & $\mathrm{Zn}$ \\
\hline $59.0-62.0$ & $<0.10$ & 0.07 & Res. \\
\hline
\end{tabular}

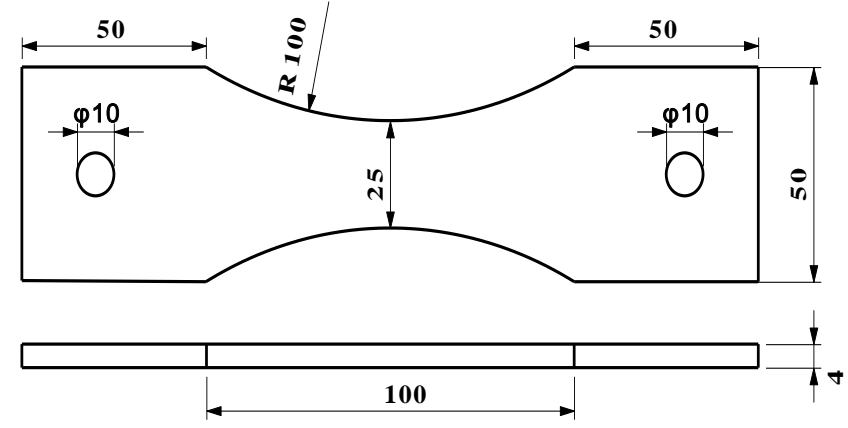

Fig. 3. Specimen Shape and Dimension.

\section{Sample Preparation}

Specimen was annealed at $837 \mathrm{~K}\left(600^{\circ} \mathrm{C}\right)$ for 4 hours to remove residual stress and was polished using sand paper \#100 - \#800 to minimize scattered ultrasonic waves.

\section{Fatigue Test}

Fatigue test was carried out using a fatigue machine with tensile test. In this test, load was acted cyclically in sine wave, where the maximum load was kept constant at $150 \mathrm{MPa}$. Stress ratio, R, a comparison between minimum and maximum stress, was 0.05 and cycle frequency was $10 \mathrm{~Hz}$. The test was carried out in room temperature. At particular number of load cycle, the test was ceased and the specimen was removed from the testing machine and then ultrasonic test was conducted. After ultrasonic test, fatigue test was continued. This procedure was carried out repeatedly until the specimen experienced fracture. In the same time, observation was performed to confirm whether fracture had occurred.

\section{Ultrasonic Method}

In this ultrasonic method, ultrasonic water immersion (UWI) procedure was used. The experimental equipment was set up as shown in Fig. 4. The test used a probe of focusing type with focus distance of $25 \mathrm{~mm}$ and nominal frequency of $15 \mathrm{MHz}$. During the test, the focus point of the probe was positioned at the lower surface of the specimen such that the first wave amplitude reached 
maximum value. To find out the effect of plastic deformation on the section area, ultrasonic test was carried out in the middle part of the specimen with interval of $5 \mathrm{~mm}$. In the area that was predicted to undergo big strain, the interval was kept $1 \mathrm{~mm}$ (14 points along the length direction and 23 points along width direction) as illustrated in Fig. 5.

Testing in this area was conducted by analyzing surface reflected wave and back wall reflected wave. Test data of ultrasonic wave were digitalized and directly acquisitioned using a computer. Digitalization was carried out at sampling rate of $0.2 \mathrm{~ns}$ and every wave datum consisted of 10,020 points of data.

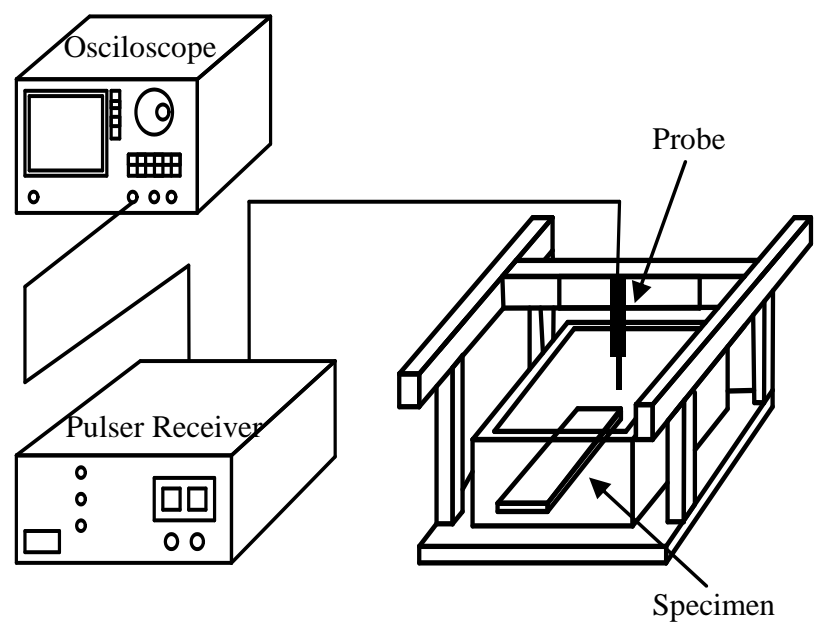

Fig. 4. Schematic Equipment of Ultrasonic Test.

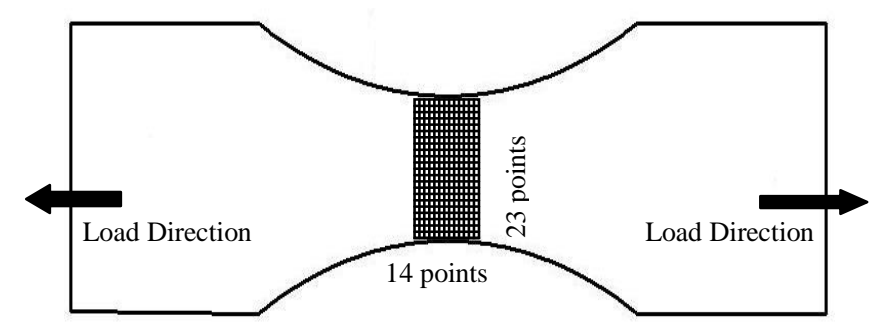

Fig. 5. Ultrasonic Testing Area.

\section{RESULTS AND DISCUSSION}

Frequency spectrum peak intensity of back wall reflected wave has been achieved for every measurement point along the middle area of the specimen as shown in Fig. 6. It can be seen that peak intensity rises drastically at the beginning of load cycle. The next peak is slightly lower than the previous one. Later the peak intensity goes down. It seems that the drastic rise of the peak intensity is attributed to large strain acting on the specimen during low-cycle fatigue test, which makes it experience large plastic deformation causing a decrease in its thickness. This thinning specimen generates high peak intensity of frequency spectrum. However, in general clear association between load cycle and peak intensity of frequency spectrum has not been identifiable.

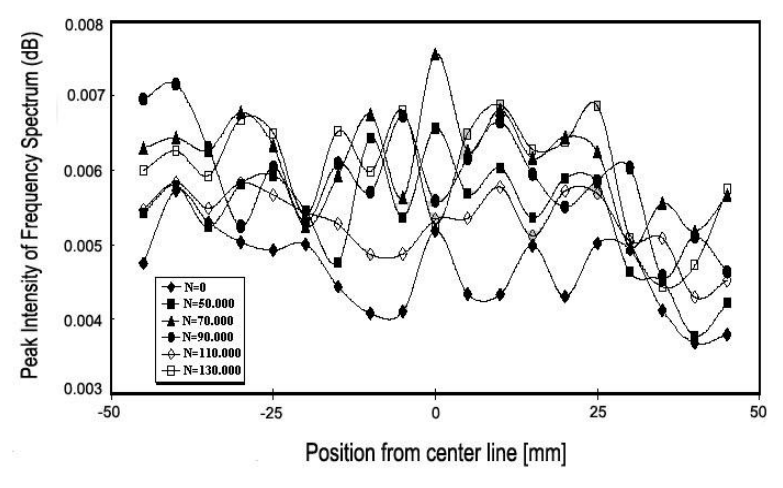

$\mathrm{N}$ : Number of Cycle

Fig. 6. Frequency Spectrum Peak Intensity along the Specimen Middle Area.

Distribution of frequency spectrum peak intensity is displayed in Fig. 7 showing that there is a pattern along the load axis. Following an increased-load cycle, a belt-shaped pattern (indicated by red color) is also apparent. Addition of the number of load cycle, this pattern does not shift. Peak intensity in this belt-shaped pattern goes higher than that of the initial condition. This phenomenon corresponds with large stress acting on the specimen at the beginning of load cycle causing plastic deformation. The belt-shaped pattern indicates the presence of slip band on the specimen [2,3]. Clearly, patterns of contour obtained from the ultrasonic test reflect the changes in the material microstructure. It was found that the first fracture was first observed at $\mathrm{N}=130,000$ and the break up occurred at $\mathrm{N}=133,900$. Since the break up started from the edge, not the surface, of the specimen, it was not identified by back wall reflected wave signal. When the number of load cycle during fracture initiation was compared with the number of load cycle causing the break up, it was found out that fatigue predominates the occurrence of fracture initiation. This indicates that fatigue degradation monitoring is essential.

Attenuation function gradient in relation to the number of load cycle at the edge and in the middle area of the specimen has also been obtained and shown in Fig. 8. After the first load, the attenuation function gradient decreases and reaches a minimum level. Following the subsequent loads, it rises gradually. At specimen break up, the gradient gets lower. This trend occurs at the edge and in the middle of the area of the specimen. 


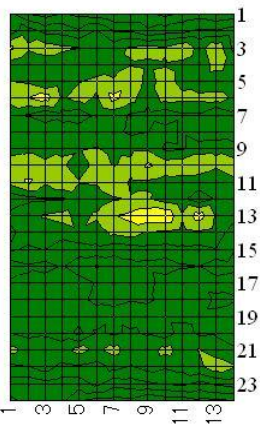

(a)

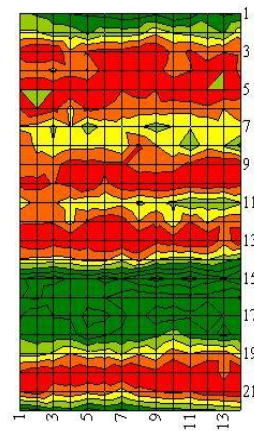

(d)

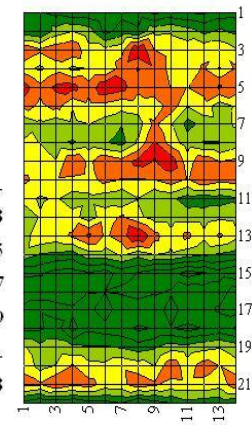

(b)

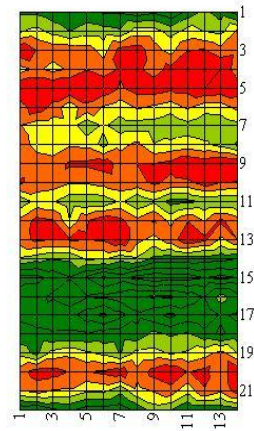

(e)

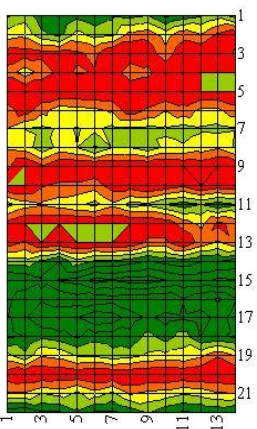

(c)

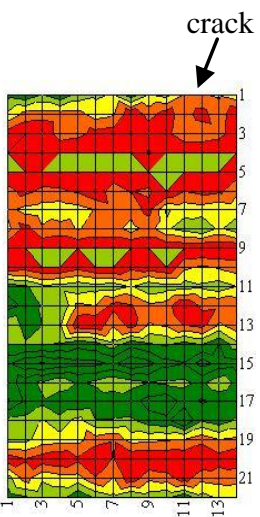

(f) \begin{tabular}{l}
$\square, 004 \quad \square 0,004-0,0045 \quad \square 0,0045-0,005$ \\
\hline
\end{tabular} $\square_{0,005-0,0055} \square_{0,0055-0,006}$

(a) $\mathrm{N}=0$, (b) $\mathrm{N}=50.000$, (c) $\mathrm{N}=70.000$, , d) $\mathrm{N}=90.000$,

(e) $\mathrm{N}=110.000$, (f) $\mathrm{N}=130.000 \mathrm{~N}$ indicates number of load cycle

Fig. 7. Distribution of Frequency Spectrum Peak Intensity

Interaction of ultrasonic wave and material resulting in attenuation change depends on two variables: dislocation and scattering by material grain boundary. When an ultrasonic wave enters to material, dislocation in material will use the energy of the wave to vibrate. Therefore, the energy of the ultrasonic wave decreases or attenuates.

At the beginning of fatigue process, large plastic deformation occurs in a very short time and causes slip band, which then increases dislocation density. This high dislocation density finally emerges to the subsurface[5] and lengthens dislocation loop. Consequently, attenuation rises. Moreover, plastic deformation also causes frequency spectrum to get higher. The increase in attenuation and frequency spectrum makes attenuation gradient lower as indicated in Fig. 8 .

In the next fatigue process, plastic deformation is relatively constant and material hardening takes place. Dislocation density decreases and mobility density slows down, which then lower the attenuation. These conditions cause attenuation gradient to increase as presented in Fig. 8.
Attenuation of ultrasonic wave corresponds with scattering by grain boundary as represented by equation [5].

$$
\alpha=a f+S D^{3} f^{4}
$$

where $\alpha$ is attenuation of ultrasonic wave, $a$ is absorption constant, $S$ is scattering coefficient, $D$ is grain diameter, and $f$ is frequency. During fatigue process, the specimen experiences elongation and thinning, which makes microstructure smaller and longer. Consequently, strain becomes higher, which increases attenuation and lowers the gradient of attenuation function. Additional experiment carried out also confirms that the shift to higher frequency also lessens gradient of attenuation function.

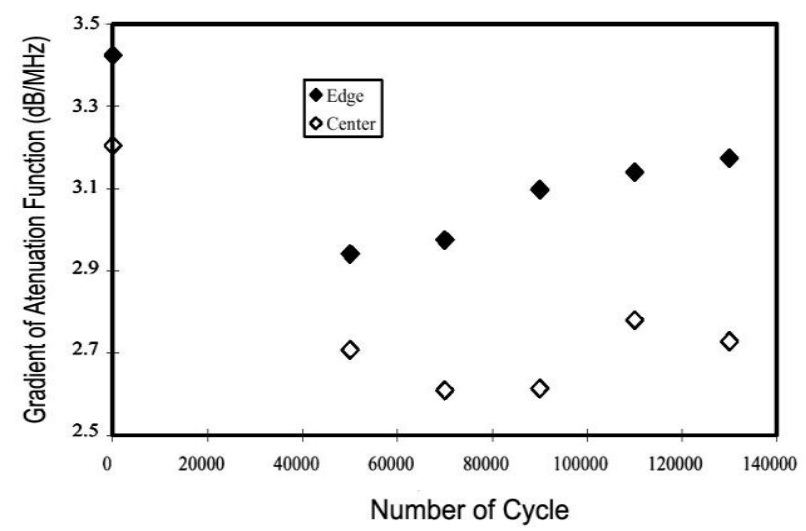

Fig. 8. Relation between Gradient of Attenuation Function and Load Cycle.

\section{CONCLUSIONS}

Ultrasonic method, which is a non-destructive test, has been applied to monitor material degradation due to low-cycle fatigue. The results show that material experiencing low-cycle fatigue may result in fracture. The test indicates that load cycle causes plastic deformation on the material tested. The pattern obtained by distribution of frequency spectrum peak intensity in the specimen reflects the occurrence of slip band in microstructure. The fracture happens at the edge of specimen. The results indicate that low-cycle fatigue is a predominant factor causing material fracture. Gradient of attenuation function decreases at initial fatigue process and then goes higher consistently. This study concludes that fatigue growth caused by low-cycle should be monitored continuously and ultrasonic method is able to monitor such material degradation. 


\section{REFERENCES}

1. L.F. Coffin, Transaction of the ASME $\mathbf{7 6}$ (1954) 931.

2. Ching, et al., NDT\&E International 32 (1999) 355.

3. Katoh, et al., Journal of the Japanese Society for Non-Destructive Inspection 50 (2001) 34.
4. Suprapedi and Toyooka, Optical Review 4 (1997) 284.

5. Hirao, et al., Acta Materialia 48 (2000) 517.

6. Anonymous, Ultrasonic Flaw Detection Method, in: Japanese Industrial Newspaper (1974) 13. 
R. Himawan, et al / Atom Indonesia Vol. 36 No. 2 (2010) 63 - 68 